\title{
Reactive Oxygen Species: A Double-Edged Sword in Reproduction
}

\author{
F. Moreira da Silva*, A. Marques and A. Chaveiro \\ University of the Azores, Department of Agrarian Sciences, Animal Reproduction - CITAA 9701-851, Angra do \\ Heroismo, Portugal
}

\begin{abstract}
Antioxidants are compounds that protect cells against the damaging effects of reactive oxygen species (ROS) such as singlet oxygen, superoxide, peroxyl radicals, hydroxyl radicals and peroxynitrite. Oxidative damage occurs to cells in vivo and in vitro from exposure to free radicals generated by exogenous agents (e.g., radiation, chemicals, hyperoxia) and endogenous processes such as normal cellular metabolism. An imbalance between antioxidants and ROS results in oxidative stress, leading to cellular damage. Oxidative stress (OS) has been linked to cancer, ageing, atherosclerosis, ischemic injury, inflammation, and neurodegenerative diseases. Under extreme oxidative conditions, or if the antioxidant protective mechanisms of cells are compromised, cellular injury and death may occur. Early mammalian embryos are susceptible to damage from reactive oxygen species, and they increase the production of oxygen free radicals when cultured in vitro. ROS generation results from mitochondria's oxidative phosphorylation. The electrons will leak from the inner mitochondrial membranes, being transferred by the oxygen molecule, resulting in an unpaired electron in the orbit. This leaking results in the generation of the superoxide molecules. ROS can also be generated by the cytoplasmic NADPH-oxidase, cytochrome p450 enzymes and the xanthine oxidoreductase enzymes. The excess of OS can have negative effects on the cellular environment and can result in impaired cellular growth in the embryo or apoptosis resulting in embryo fragmentation. Similarly to what happens in females, oxidative energy production in males is inevitably associated with the generation of ROS excessive concentrations of which can lead to cellular pathology. It has been established that ROS can function as signaling molecules and evidence is emerging that sperm may generate low and controlled concentrations of ROS, specifically $\mathrm{O}_{2}-\mathrm{H}_{2} \mathrm{O}_{2}$, as well as other species such as nitric oxide (NO), which, in his turn, act to mediate the processes of capacitation, hyperactivation and acrosome reaction crucial to the acquisition of fertilizing ability. In the present review the most important antioxidants and their mechanisms of action related to animal reproduction, are discussed.
\end{abstract}

Keywords: ROS, oxidative stress, animal reproduction.

\section{INTRODUCTION}

The term antioxidant, also so called antioxygen, in the 19 th and early 20th century, has been referred specifically to a chemical that prevented the consumption of molecular oxygen. Antioxidants were then subjected of extensive research as they protect cells against the damaging effects of reactive oxygen species (ROS), such as singlet oxygen, superoxide, peroxyl radicals, hydroxyl radicals and peroxynitrite. An imbalance between antioxidants and ROS results in an oxidative stress (OS), leading to cellular damage. OS has been linked to cancer, ageing, atherosclerosis, ischemic injury, inflammation and neurodegenerative diseases $[1,2]$.

There are two types of antioxidants: enzymatic and nonenzymatic:

Enzymatic antioxidants are also known as natural antioxidants, act by neutralizing excessive ROS, and prevent it from damaging the cellular structure. They are composed of catalase, glutathione redutase, glutathione peroxidase and superoxide dismutase, which causes reduction of hydrogen

*Address correspondence to this author at the University of the Azores, Department of Agrarian Sciences, Animal Reproduction - CITAA 9701851, Angra do Heroísmo, Portugal; Tel: +351 295402 200; Fax: +351 295 402 205; E-mail: jsilva@uac.pt peroxide to water and alcohol [3]. Superoxide dismutase and glutathione peroxidase are natural antioxidants present in organisms which eliminate some ROS and glutathione peroxidase catalyzes the reduction of peroxide by oxidizing glutathione (GSH) to oxidized glutathione [4].

Non-enzymatic antioxidants are also known as synthetic antioxidants or dietary supplements [3]. The body's complex antioxidants system is influenced by dietary intake of antioxidant vitamins and minerals such as vitamin $\mathrm{C}$, vitamin E, selenium, zinc, taurin, hypotaurin, glutathione, beta carotene and carotene [5]. Vitamins $\mathrm{C}$ and $\mathrm{E}$ are not produced in the body but must be obtained through food. Glutathione is produced by the body, but levels of this antioxidant decline with age [6]. Research into how vitamin E ( $\alpha$-tocopherol and derivatives) prevents the process of lipid peroxidation, as antioxidants reduce agents that break oxidative chain reactions, often by scavenging reactive oxygen species before they can cause damage to the cells [7]. This vitamin, the predominant lipid-soluble antioxidant in animal cells, protects cells from oxygen radicals in vivo and in vitro, and is believed to be the primary free radical scavenger in mammalian cell membranes [2]. Vitamin E has been considered as a natural antioxidant that reacts with soluble free radicals in lipids membranes. Its active place locates in the group $\mathrm{OH}$ in the position 6 of the phenol ring. The oxidative attack that polyunsaturated greasy acids suffer by the radical $\mathrm{OH} \bullet$ and $\mathrm{O}_{2} \bullet$, produces radical peroxil ( $\mathrm{ROO} \bullet$ ) 
[8]. This vitamin protects lipids from peroxidation, being oxidized to tocopheryl quinone or into tocopheroxyl free radical. In both cases, it is reduced by ascorbate (Vitamin C), which is afterwards oxidized into dehydroascorbate or ascorbate free radical.

Enzymatic and non-enzymatic antioxidants rapidly scavenge the attacking radical and thereby terminate its destructive pathways. This mechanism presumes that the resulting antioxidant derived radical is a "harmless" one, i.e., the reactivity of the antioxidant radical toward typical biomolecules must be low [9]. Moreover, the antioxidant $\beta$ Mercaptoethanol ( $\beta$-ME) is a low weight thiol compound with a reducing power, interacting directly with some oxidized radicals and which can chelate metallic ions. It protects cysteine, a precursor of GSH, from oxidation into cystine and increases its entry into the cell, which is known to trigger GSH synthesis [10].

\section{OXIDATIVE STRESS}

Sies [11] defined "oxidative stress" as "a disturbance in the prooxidant-antioxidant balance in favor of the former". This definition does not consider that free radical generation may easily be of benefit. Therefore an updated definition of oxidative stress could be "severe disturbance in the prooxidants-antioxidants balance in favor of the former, thus leading to a potential damage to the cells and organs". Essentially, "oxidative stress" occurs when the formation of bioactive oxidation products greatly overwhelms the capacity of endogenous cellular antioxidant defence system. The resulting damage to cells and organs may activate and/or accelerate disease processes [12].

By definition a free radical is any atom (e.g. oxygen, nitrogen) with at least one unpaired electron in the outermost shell, which is capable of independent existence. A free radical is easily formed when a covalent bond between entities is broken and one electron remains with each newly formed atom [13]. Free radical species are highly reactive and unstable, only becoming stables by acquiring electrons from, lipids, proteins, nucleic acids, carbohydrates or any nearby molecule and thus causing a cascade of damage and disease [5]. There are two key types of free radical species: Reactive oxygen species and reactive nitrogen species.

Any free radical involving oxygen can be referred to as a ROS. Oxygen centred free radicals contain two unpaired electrons in the outer shell. When free radicals steal an electron from a surrounding compound or molecule a new free radical is formed in its place [14]. As referred to above, the most common ROS include: the superoxide anion $\left(\mathrm{O}_{2}{ }^{-}\right)$, the hydroxyl radical $(\mathrm{OH} \bullet]$, singlet oxygen $\left({ }^{1} \mathrm{O}_{2}\right)$, and hydrogen peroxide $\left(\mathrm{H}_{2} \mathrm{O}_{2}\right]$. Superoxide anions are formed when oxygen $\left(\mathrm{O}_{2}\right)$ acquires an additional electron, leaving the molecule with only one unpaired electron. Within the mitochondria $\mathrm{O}_{2}{ }^{-} \bullet$ is continuously being formed. The rate of formation depends on the amount of oxygen flowing through the mitochondria at any given time. Hydroxyl radicals are short-lived, but are the most damaging radicals within the body. This type of free radical can be formed from $\mathrm{O}_{2}{ }^{-}$and $\mathrm{H}_{2} \mathrm{O}_{2}$ via the Harber-Weiss reaction $\left(\mathrm{O}_{2} \bullet^{-}+\mathrm{H}_{2} \mathrm{O}_{2} \rightarrow \mathrm{OH} \bullet+\right.$ $\left.\mathrm{OH}^{-}+\mathrm{O}_{2} \bullet{ }^{-}\right][15]$.

Hydrogen peroxide is produced in vivo by many reactions, either being converted to the highly damaging hydroxyl radical or catalyzed and excreted harmlessly as water [14]. The principal problem is that $\mathrm{H}_{2} \mathrm{O}_{2}$ easily crosses cellular membranes while receiving one more electron, normally originating from iron or copper, gives rise to the hydroxyl radical. This radical represents the most reactive oxygen species, as it needs only one more electron to be stabilized. Cells living under aerobic conditions constantly face the oxygen $\left(\mathrm{O}_{2}\right)$ paradox: $\mathrm{O}_{2}$ is required to support life, but its metabolites such as reactive oxygen species (ROS) can modify cell functions, endanger cell survival, or both [16].

ROS have been associated with several diseases [17, 18], playing a particular role in the female reproductive tract on ovaries [19, 20], and even on embryos [21]. These compounds are also implicated in the reproductive functions such as ovarian steroidogenesis, oocyte maturation, corpus luteal function and luteolysis [19], thus being related to female fertility.

Concerning nitric oxide (NO), which is synthesized during the enzymatic conversion of $\mathrm{L}$-arginine to L-citrulline by nitric oxide synthase [22-24], which is a highly reactive free radical with an unpaired electron, that could damage carbohydrates, proteins, nucleotides and lipids and, together with other inflammatory mediators [23]. NO relaxes arterial and venous smooth muscles and, less strongly, inhibits platelet aggregation and adhesion. NO donors, acting as vasodilating agents, are therefore a possible therapeutic approach [25]. NO acts in a variety of tissues to regulate a diverse range of physiological processes, being, however, toxic in excess $[5,26]$.

Reactive nitrogen species have been associated with asthma, ischemic/reperfusion injury, septic shock and atherosclerosis [27-30]. The most common examples of reactive nitrogen species are the above referred NO and nitrogen dioxide [5, 31]. NO is synthesized by the enzyme NO synthase. In mammals there are three types of nitric oxide synthase enzymes involving endothelial NO synthase (NO synhase 3), neuronal NO synthase (NO synthase1) and inducible $\mathrm{NO}$ synthase (NO synthase 2). Inducible NO synthase is triggered by cytokines such as, interleukin-1, and TNF- $\alpha$ and lipopolysaccharides. Nitric oxide synthase is expressed in reproductive cells like granulosa cells, thecal cells, and surface of oocyte during the follicular development. In case of diseases, inducible NO synthase might play an important role in NO production. In most organs, inducible NO synthase is expressed only in response to immunological stimuli [32].

\section{OXIDATIVE STRESS IN FEMALE REPRODUCTION}

Is a given fact that perfectly normal and healthy individuals, even under basal conditions, produce ROS through their aerobic metabolism. Therefore cells have developed a wide range of antioxidant mechanisms to limit the production of ROS, inactivate them and repair cell damage $[5,33]$.

In a healthy body, ROS and antioxidants remain in balance. Nevertheless, when this balance is disrupted towards an excess of ROS, oxidative stress occurs. ROS are a double-edged sword: they have a role in pathological processes involving the female reproductive tract, but also 
can serve as key signal molecules in physiological processes [3].

Lately, Agarwal and coworkers [33] reported that OS has an important role in the normal functioning of the female reproductive system and in the pathogenesis of female infertility, influencing the entire reproductive span of a females' life. ROS can modulate cellular functions, and OS can impair the intracellular environment resulting in diseased cells and endangered cell survival. ROS can affect a variety of physiological functions in the reproductive tract, and excessive levels can result in pathologies affecting female reproduction. The oxidant status can influence early embryo development by modifying the key transition factors and hence modifying gene expression [34].

De Bruin and collaborators [35] suggested that the agerelated decline in fertility is influenced by this form of stress. During gestation OS plays a role in the initiation of preterm labor [36, 37] and during normal parturition [38, 39], assuring ovulation, ovarian steroidogenesis, oocyte maturation, blastocyst formation, luteolysis and luteal maintenance in pregnancy [20,40]. Concentrations of ROS may play a key role both in the implantation and fertilization of oocytes [41]. Oxidative stress operates in follicular development, normal cycling ovaries, and cyclical endometrial changes. Several probes of oxidative stress such as superoxide dismutase, $\mathrm{Cu}-\mathrm{Zn}$ superoxide dismutase, glutathione peroxidase, $\gamma$ glutamil synthetase and lipid peroxides have been investigated by immunohistochemical location [40, 42]. Suzuki et al. [20] founded a delicate balance between ROS and antioxidant enzymes in the ovarian tissues in all follicular stages which has been examined for superoxide dismutase expression including primordial, primary, preantral, nondominant antral follicles, dominant follicles and athretic follicles. On the other hand, the pathological effects are exerted by various mechanisms including lipid damage, inhibition of protein synthesis and depletion of ATP [43]. OS is also involved in the etiology of defective embryo development and seems responsible for numerous types of embryo damage. $\mathrm{ROS}$ such as $\mathrm{O}_{2}{ }^{-}$are able to diffuse and pass through cell membranes and alter most types of cellular molecules such as lipids, proteins and nucleic acids. The consequences are multiple and include mitochondrial alterations, embryo cell block, ATP depletion and apoptosis. ROS also induce lipid peroxidations with related effects in cell's division, metabolite transport and mitochondrial dysfunction. The 2-cell block observed in mouse embryos is associated with a rise in lipid peroxides $[44,45]$. ROS can induce protein sulphydryl oxidation and disulphide formation. With oxidative stress, the rates of disulphide bonds and mixed disulphide formations increasing within the cell. As a consequence, inactivation of enzymes such as glyceraldehydes 3-phosphate dehydrogenase $(\mathrm{G} 3 \mathrm{PDH})$ can occur [46]. It was also reported that ROS induce DNA strand breaks [47]: a four-fold increase in the nuclear DNA fragmentation rate is observed after spermatozoa are exposed to ROS [48]. Such nuclear DNA lesions are involved in embryo development arrest observed in vitro. OS induces mitochondrial damage $[49,50]$. Mitochondrial DNA (mtDNA) is especially susceptible to mutation because of its lack of histones which normally quench ROS [49, 50]. During OS, mtDNA mutation is fourfold more frequent than nuclear DNA mutations. This way, defective embryo mtDNA may induce metabolic dysfunction and, consequently, disturb embryo development; arrest of embryo development observed in vitro is associated with dysfunction of mitochondria. The consequences of these alterations are multiple, and include embryo development retardation and arrest, metabolic dysfunctions and possibly also apoptosis. ROS have been implicated in the impaired development of mammalian embryos in vitro [51, 52]. The 2-cell embryo block observed in mouse embryos is associated with a rise in $\operatorname{ROS}[44,45]$, which is only observed after culture in vitro. No such effect is observed in embryos collected in vivo [45]. Deleterious effects of ROS during oocyte maturation may alter embryo development [53]. ATP depletion occurs in cells via inactivation of glycerol-3-dehydrogenase $\left(\mathrm{G}_{3} \mathrm{PDH}\right)$ and/or inactivation of glycolitic and mitochondrial pathways [54]. OS induces consumption of reducing equivalents such as GSH. Glutathione reductase (GR) activity allows the GSH endogenous pool to be maintained. GR is NADPH dependent and the main source of NADPH in the monophosphate shunt (pentose phosphate pathway). Consequently, oxidative stress, via competitive consumption of reducing equivalents, can interfere with important metabolic functions and divert glucose from other pathways by inducing the monophosphate shunt.

Accumulation of superoxide radicals and a decline in superoxide dismutase (SOD) levels are involved in apoptotic cell death, whereas antioxidants including SOD can inhibit apoptosis. $\mathrm{H}_{2} \mathrm{O}_{2}$ is a mediator of apoptosis in blastocysts [55]. The appearance of cytoplasmic fragments in blastocysts seems to be related to apoptosis [56]. A direct relationship was also observed between increased $\mathrm{H}_{2} \mathrm{O}_{2}$ concentration and apoptosis in human fragmented embryos [57]. Fragmentation in mouse and human embryos occurs just before the time of in vitro block; such fragmentation may be a mechanism to regulate the nucleocytoplasmic ratio in blastomeres, and/or a protective mechanism against damage induced by oxidative stress.

In assisted reproduction technology (ART), the freezethaw process makes cells more sensitive to ROS. Furthermore, the freeze-thaw process reduces GSH concentrations by $78 \%$ and SOD activity by $50 \%$ in bovine spermatozoa [58]. The increase in lipid peroxidation observed after sperm cryopreservation is due to a loss of SOD activity [59], which strongly suggests that oxidative stress occurs during and/or after the cycle of freeze-thaw. This way partly explains the observed deleterious effect of cryopreservation on gamete/embryo viability. Modifications of membrane lipids related to ROS and the resulting spatial modifications of membrane structures may clearly lead to cryodamage.

ROS play a physiological role in gametes and embryos, they are implicated in the control of capacitation, acrosomal reaction [60] and fertilization [61] processes, being probably implicated in regulating the speed of pre-implantation embryo development [62].

Embryos may also have different sensitivities to ROS at different developmental stages. For example, it has been observed [63] that 9 to 16 cell bovine embryos are more resistant to exogenous $\mathrm{H}_{2} \mathrm{O}_{2}$ than zygotes and blastocysts. 
These different sensitivities are due to variations in defense mechanism thresholds.

\section{OXIDATIVE STRESS IN MALE REPRODUCTION}

Similarly to what happens in females, oxidative energy production in males is inevitably associated with the generation of reactive oxygen species (ROS) in which excessive concentrations can lead to cellular pathology. It has been established that ROS can function as signaling molecules and evidence is emerging [64] that sperm may generate low and controlled concentrations of ROS, specifically $\mathrm{O}_{2}-\mathrm{H}_{2} \mathrm{O}_{2}$, as well as other species such as nitric oxide (NO), which act to mediate the processes of capacitation, hyperactivation and acrosome reaction crucial to the acquisition of fertilizing ability [64]. Mild oxidative conditions resulting from low concentrations of ROS may also stimulate sperm-binding to the zona pellucida [65]. Although the precise nature and concentration of ROS varies with experimental conditions, data are converging to describe this event as oxidative or redox regulated. The balance between the timing and location of ROS production and scavenging must be respected if sperm function is not to be compromised.

Although the precise mechanism by which ROS operate as transduction molecules in sperm are yet to be elucidated, a scheme of action can be proposed. Sperm in the basal state generate low net concentrations of ROS. Incubation in capacitating conditions stimulates generation of superoxide anion $\left(\mathrm{O}^{2-}\right)$ through an oxidase not yet characterized. $\mathrm{O}^{2-}$ and hydrogen peroxide $\left(\mathrm{H}_{2} \mathrm{O}_{2}\right)$ formed by dismutation of $\mathrm{O}_{2}{ }^{2-}$ can induce sperm capacitation, which is also promoted by NO. The targets for this ROS are unknown but there is a redoxregulated increase in tyrosine phosphorylation of specific proteins during capacitation. Induction of the $A R$ in capacitated sperm stimulates $\mathrm{O}_{2}$ production which causes release of unesterified fatty acids from the plasma membrane of these cells. The ROS target for AR induction is unknown but again tyrosine phosphorylation of specific proteins is involved [64].

The mechanism of ROS production by sperm is still unclear. An NADPH oxidase generating $\mathrm{O}_{2^{-}}$, similar to that found in phagocytic leucocytes has been proposed [66], based on observations of the effect of NADPH addition to purified sperm using a lucigenin-based chemiluminescence assay. However, NADPH was shown not to stimulate extracellular $\mathrm{O}_{2-}$ production in sperm as detected by 2-methyl-6-(rho-methoxyphenyl)1-3,7 dihydroimidazo [1,2-a] pyrazin-3-one chemiluminescence whereas addition of biological fluids inducing capacitation (e.g. follicular fluid) did so [67].

McLeod [68] first demonstrated that incubation in a oxygen rich environment was detrimental to human spermatozoa, decreasing motility and viability. Since then, many reports have associated ROS with impaired sperm function including decreased motility, abnormal morphology, and decreased sperm-egg penetration [65, 69-72]. Spermatozoa are highly susceptible to damage by excess concentrations of ROS due to the high content of polyunsaturated fatty acids within their plasma membrane and, although conventional basic semen characteristics other than motility are not obviously influenced by the oxidative state of semen [66], such damage may underlie several aspects of male infertility. Increased lipid peroxidation and altered membrane function can render sperm dysfunctional through impaired metabolism, motility, acrosome reaction reactivity and fusogenic capacity as well as oxidative damage to sperm DNA [73]. Patients with asthenozoospermia (impaired sperm motility) have increased concentrations of ROS in seminal plasma and increased ROS-mediated damage of sperm membranes, although whether oxidative damage occurs in the testes, epididymis or semen is still uncertain. Abnormal sperm, characterized by retention of excess residual cytoplasm as a result of defective spermatogenesis, are a source of ROS in semen [74], but t is generally accepted that contaminating leukocytes are the primary source of ROS in unpurified sperm suspensions [75-78].

Semen possesses a variety of antioxidant scavenger defence mechanisms including catalase, uric acid, taurine, thiols, ascorbic acid and alpha-tocopherol but principally superoxide dismutase (SOD) and the glutathione-peroxidasereductase system $[79,80]$. Extracellular SOD binds to the neck region of a subgroup of sperm which retain motility longer than those without bound SOD and both the proportion of sperm binding SOD and total SOD activity vary widely among samples; however, any reproductive significance of this remains unknown. Despite this range of defences apparently available, it has been suggested that mature sperm may yet be inadequately protected due to their high concentration of membrane unsaturated lipids together with a relative paucity of enzymes such as SOD due to the virtual absence of cytoplasm [81]. This is partly compensated for by the powerful antioxidant system present in seminal plasma which, in contrast to other biological fluids, contains significant concentrations of SOD, xanthine oxidase, nitric oxide, catalase, glutathione peroxidase, plus ascorbic acid, thiols, uric acid, alpha-tocopherol and a high level of glutathione [82-84]. Moreover, sperm do retain functional concentrations of antioxidant enzymes despite their sparsecytoplasm $[85,86]$. Sperm defences need only ensure their survival until they achieve fertilization; they are then removed from the female reproductive tract. However, it is crucial that this balance be struck as inadequate defences lead to premature loss of function and thus impair fertility.

\section{OXIDATIVE STRESS IN ASSISTED REPRODUCTIVE TECHNIQUES}

Assisted reproductive techniques (ART) involve the direct manipulation of the oocytes, sperm or embryos outside the body, to establish a pregnancy.

The follicular fluid microenvironment has an important role in determining the quality of the oocyte, which has an impact in the fertilization rate and the embryo quality. Markers for oxidative stress have been localized in the follicular fluid in patients undergoing IVF/embryo transfer (ET) $[42,87,88]$. Low intrafollicular oxygenation has been related with decreased oocyte developmental potential as reflected by increasing frequency of oocyte cytoplasmic defects, impaired cleavage and abnormal chromosomal segregation in oocytes from poorly vascularised follicles [89]. ROS may be responsible for generating increased embryo fragmentation, resulting from increased apoptosis 
[57]. Thus increasing ROS levels is not favourable to embryo growth and result in deficient development.

Increase in the total antioxidant capacity was observed in follicular fluid of oocytes that were successfully fertilized, lower levels were associated with increased viability of the embryos until the time of transfer, being, therefore, the total antioxidant capacity a predictive of decreased fertilization potential and the fertilization potential decreased with decreasing concentrations of total antioxidants. Therefore, lower total antioxidant capacity is predictive of decreased fertilization potential [88].

Oxidative stress in follicular fluid from women undergoing IVF was inversely correlated with the women's age [90] in which the slope was found to positively correlate with maximal serum estradiol levels, number of mature oocytes and number of cleaved embryos and inversely with the number of gonadotropin ampoules used. The pregnancy rate achieved was $28 \%$ and all pregnancies occurred when the thermochemiluminescence amplitude was small. This is in agreement with another study that reported minimal levels of OS were necessary for achieving pregnancy [89]. Follicular fluid ROS and lipid peroxidation levels may be markers for success with IVF.

IVF culture media may be the exogenous site of ROS generation affecting the oocytes and the pre-implantation embryo [3]. Harvey et al. [91] suggested that there are some specific events in embryo development associated with a shift in the redox state, that may have a causative role in sperm mediated oocyte activation, embryonic hatching from the zona pellucida and embryonic genome activation. In vivo, fertilization and embryo development occurs in an environment of low oxygen tension [92]. During ART, it is important to avoid conditions that promote ROS generation and expose embryos and gametes to ROS [3].

As known amino acids added to the IVF media also have antioxidant properties. The benefits of adding such substances as ascorbate during cryopreservation reduces hydrogen peroxide levels and thus the oxidative distress in mammalian embryos [93], resulting in a better embryo development and improving the blastocyst development rates.

\section{CONCLUSION}

From the above exposed the role of antioxidants in the reproductive mechanisms still quite controversial, besides the number of published papers studying their roles in male and female reproduction in the last years increased more and more. The effects of ROS in terms of the oocyte, spermatozoa, fertilization, embryo development and gestation show that different concentrations of ROS can lead different reactions in cell's metabolism. More research must be implemented using different antioxidants in different concentrations, with elimination of various factors leading to bias.

\section{ACKNOWLEDGMENT}

Research developed in the field of antioxidants was supported by the DRCT (Regional Government of the Azores), grant number M2.1.1///004/2005.

\section{REFERENCES}

[1] Buhler DR, Miranda C. Antioxidant activities of flavonoids Oregon State University: USA 2000.

[2] Olson SE, Seidel GE, Jr. Culture of in vitro-produced bovine embryos with vitamin $\mathrm{E}$ improves development in vitro and after transfer to recipients. Biol Reprod 2000; 62(2): 248-52.

[3] Agarwal A, Said TM. Oxidative stress, DNA damage and apoptosis in male infertility: a clinical approach. BJU Int 2005; 95(4): 503-7.

[4] Mignone J. Chemical modifications that lead to protein degradation biochemistry. 2002; Available from: http://mercury.chem.pitt.edu/ $\sim$ rob/chem2810/present281/james_mignone.ppt

[5] Pierce JD, Cackler AB, Arnett MG. Why should you care about free radicals? RN 2004; 67(1): 38-42; quiz 3.

[6] Packer L. The antioxidant miracle. New York: John Wiley \& Sons 1999.

[7] Wolf G. The discovery of the antioxidant function of vitamin E: the contribution of Henry A. Mattill. J Nutr 2005; 135(3): 363-6.

[8] Rodríguez GP. Funciones de la vitamina E en la nutrición humana. Rev Cubana Aliment Nutr 1997; 11(1): 46-57.

[9] Kirsch M, De Groot H. NAD $(\mathrm{P}) \mathrm{H}$, a directly operating antioxidant? FASEB J 2001; 15(9): 1569-74.

[10] Feugang JM, de Roover R, Moens A, et al. Addition of betamercaptoethanol or Trolox at the morula/blastocyst stage improves the quality of bovine blastocysts and prevents induction of apoptosis and degeneration by prooxidant agents. Theriogenology 2004; 61(1): 71-90.

[11] Sies H. Oxidative stress: introdutory remarks. In: Sies H, Ed. London: Academic Press 1985; pp. 1-8.

[12] Basu S. Fatty acid oxidation and isoprostanes: Oxidative strain and oxidative stress. Prostaglandins, Leukotrienes and Essential Fatty Acids. The Ninth Fatty Acids and Cell Signalling Meeting (FACS09), 2010; vol. 82(4-6): pp. 219-25.

[13] Karlsson JO. Introduction to nutraology and radical formation. In: Antioxidants and exercise. Illinois: Human Kinetics Press 1997; pp. $1-143$.

[14] Goldfarb AH. Nutritional antioxidants as therapeutic and preventive modalities in exercise-induced muscle damage. Appl Physiol Nutr Metab 1999; 24(3): 249-66.

[15] Vieira AJSC. Radicais oxidantes: da química à biologia. Química 2006; 100: 66-71.

[16] Ashok BT, Ali R. Aging research in India. Exp Gerontol 2003; 38(6): 597-603.

[17] Gibson GE, Huang HM. Mitochondrial enzymes and endoplasmic reticulum calcium stores as targets of oxidative stress in neurodegenerative diseases. J Bioenerg Biomembr 2004; 36(4): $335-40$.

[18] Madamanchi NR, Vendrov A, Runge MS. Oxidative stress and vascular disease. Arterioscler Thromb Vasc Biol 2005; 25(1): 2938.

[19] Behrman HR, Kodaman PH, Preston SL, et al. Oxidative stress and the ovary. J Soc Gynecol Investig 2001; 8(1): S40-2.

[20] Suzuki T, Sugino N, Fukaya T, et al. Superoxide dismutase in normal cycling human ovaries: immunohistochemical localization and characterization. Fertil Steril 1999; 72(4): 720-6.

[21] Guerin P, El Mouatassim S, Menezo Y. Oxidative stress and protection against reactive oxygen species in the pre-implantation embryo and its surroundings. Hum Reprod Update 2001; 7(2): 17589.

[22] Vega M, Johnson MC, Diaz HA, et al. Regulation of human luteal steroidogenesis in vitro by nitric oxide. Endocrine 1998; 8(2): 18591.

[23] Dong M, Shi Y, Cheng Q, et al. Increased nitric oxide in peritoneal fluid from women with idiopathic infertility and endometriosis. J Reprod Med 2001; 46(10): 887-91.

[24] Rosselli M, Keller PJ, Dubey RK. Role of nitric oxide in the biology, physiology and pathophysiology of reproduction. Hum Reprod Update 1998; 4(1): 3-24.

[25] Ohl J, Lefebvre-Maunoury C, Wittemer C, et al. Nitric oxide donors for patients undergoing IVF. A prospective, double-blind, randomized, placebo-controlled trial. Hum Reprod 2002; 17(10): 2615-20.

[26] Osborn BH, Haney AF, Misukonis MA, et al. Inducible nitric oxide synthase expression by peritoneal macrophages in endometriosisassociated infertility. Fertil Steril 2002; 77(1): 46-51. 
[27] Reynaert NL, Ckless K, Wouters EF, et al. Nitric oxide and redox signaling in allergic airway inflammation. Antioxid Redox Signal 2005; 7(1-2): 129-43.

[28] Schulman IH, Zhou MS, Raij L. Nitric oxide, angiotensin II, and reactive oxygen species in hypertension and atherogenesis. Curr Hypertens Rep 2005; 7(1): 61-7.

[29] Hsieh YY, Chang CC, Tsai FJ, et al. Glutathione S-transferase M1*null genotype but not myeloperoxidase promoter G-463A polymorphism is associated with higher susceptibility to endometriosis. Mol Hum Reprod 2004; 10(10): 713-7.

[30] Schrier RW, Wang W. Acute renal failure and sepsis. N Engl J Med 2004; 351(2): 159-69.

[31] Van Langendonckt A, Casanas-Roux F, Donnez J. Oxidative stress and peritoneal endometriosis. Fertil Steril 2002; 77(5): 861-70.

[32] Lee KS, Joo BS, Na YJ, et al. Relationships between concentrations of tumor necrosis factor-alpha and nitric oxide in follicular fluid and oocyte quality. J Assist Reprod Genet 2000; 17(4): 222-8.

[33] Agarwal A, Allamaneni SS. Role of free radicals in female reproductive diseases and assisted reproduction. Reprod Biomed Online 2004; 9(3): 338-47.

[34] Dennery PA. Role of redox in fetal development and neonatal diseases. Antioxid Redox Signal 2004; 6(1): 147-53.

[35] de Bruin JP, Dorland M, Spek ER, et al. Ultrastructure of the resting ovarian follicle pool in healthy young women. Biol Reprod 2002; 66(4): 1151-60

[36] Wall PD, Pressman EK, Woods JR, Jr. Preterm premature rupture of the membranes and antioxidants: the free radical connection. $\mathrm{J}$ Perinat Med 2002; 30(6): 447-57.

[37] Pressman EK, Cavanaugh JL, Mingione M, et al. Effects of maternal antioxidant supplementation on maternal and fetal antioxidant levels: a randomized, double-blind study. Am J Obstet Gynecol 2003; 189(6): 1720-5.

[38] Fainaru O, Almog B, Pinchuk I, et al. Active labour is associated with increased oxidisibility of serum lipids ex vivo. BJOG 2002; 109(8): 938-41.

[39] Mocatta TJ, Winterbourn CC, Inder TE, et al. The effect of gestational age and labour on markers of lipid and protein oxidation in cord plasma. Free Radic Res 2004; 38(2): 185-91.

[40] Sugino N, Takiguchi S, Kashida S, et al. Superoxide dismutase expression in the human corpus luteum during the menstrual cycle and in early pregnancy. Mol Hum Reprod 2000; 6(1): 19-25.

[41] Sharma RK, Agarwal A. Role of reactive oxygen species in gynecologic diseases. Reprod Med Biol 2004; 3(4): 177-99.

[42] Attaran M, Pasqualotto E, Falcone T, et al. The effect of follicular fluid reactive oxygen species on the outcome of in vitro fertilization. Int J Fertil Womens Med 2000; 45(5): 314-20.

[43] Ray SD, Lam TS, Rotollo JA, et al. Oxidative stress is the master operator of drug and chemically-induced programmed and unprogrammed cell death: Implications of natural antioxidants in vivo. Biofactors 2004; 21(1-4): 223-32.

[44] Nasr-Esfahani MH, Aitken JR, Johnson MH. Hydrogen peroxide levels in mouse oocytes and early cleavage stage embryos developed in vitro or in vivo. Development 1990; 109(2): 501-7.

[45] Noda Y, Matsumoto H, Umaoka Y, et al. Involvement of superoxide radicals in the mouse two-cell block. Mol Reprod Dev 1991; 28(4): 356-60.

[46] Halliwell B, Gutteridge JMC. The chemistry of oxygen radicals and other derived species. In: Halliwell B, Gutteridge JMC, Eds. Free radicals in biology and medicine. $2^{\text {nd }}$ ed. Oxford: Clarendon Press 1989; pp. 22-85.

[47] Munné S, Estop A. Superoxide anion increases after sperm storage and produces chromosome abnormalities. Biol Reprod 1991; 44: 681-7.

[48] Lopes S, Jurisicova A, Sun JG, et al. Reactive oxygen species: potential cause for DNA fragmentation in human spermatozoa. Hum Reprod 1998; 13(4): 896-900.

[49] Richter C, Gogvadze V, Laffranchi R, et al. Oxidants in mitochondria: from physiology to diseases. Biochim Biophys Acta 1995; 1271(1): 67-74.

[50] Kowaltowski AJ, Vercesi AE. Mitochondrial damage induced by conditions of oxidative stress. Free Radic Biol Med 1999; 26(3-4): 463-71.

[51] Marques A, Antunes G, Santos P, et al. Effect of alfa-tocopherol on in vitro culture of bovine embryos. Can J Anim Sci 2008; 87: 53942.
[52] Marques A, Santos P, Antunes G, et al. Effect of alpha-tocopherol on in vitro maturation of bovine cumulus-oocyte complexes. Can J Anim Sci 2008; 88(3): 463-7.

[53] Blondin P, Coenen K, Sirard MA. The impact of reactive oxygen species on bovine sperm fertilizing ability and oocyte maturation. J Androl 1997; 18(4): 454-60.

[54] Hyslop PA, Hinshaw DB, Halsey Jr WA, et al. The glycolytic and mitochondrial pathways of ADP phosphorylation are major intracellular targets inactivated by hydrogen peroxide. J Biol Chem 1988; 263: 1665-75.

[55] Pierce GB, Parchment RE, Lewellyn AL. Hydrogen peroxide as a mediator of programmed cell death in the blastocyst. Differentiation 1991; 46(3): 181-6.

[56] Yang MY, Rajamahendran R. Apoptosis in bovine oocytes and in preimplantation embryos: the role of Bcl-2 and Bax genes. Biol Reprod 1999; 60(1): 190(abstract).

[57] Yang HW, Hwang KJ, Kwon HC, et al. Detection of reactive oxygen species (ROS) and apoptosis in human fragmented embryos. Hum Reprod 1998; 13(4): 998-1002.

[58] Bilodeau JF, Chatterjee S, Sirard MA. Cryopreservation of bovine semen decreases antioxidant defenses in spermatozoa. Biol Reprod 1999; 60(1): 102.

[59] Alvarez JG, Storey BT. Evidence for increased lipid peroxidative damage and loss of superoxide dismutase activity as a mode of sublethal cryodamage to human sperm during cryopreservation. J Androl 1992; 13(3): 232-41

[60] de Lamirande E, Tsai C, Harakat A, et al. Involvement of reactive oxygen species in human sperm arcosome reaction induced by A23187, lysophosphatidylcholine, and biological fluid ultrafiltrates. J Androl 1998; 19(5): 585-94.

[61] Miesel R, Drzejczak PJ, Kurpisz M. Oxidative stress during the interaction of gametes. Biol Reprod 1993; 49(5): 918-23.

[62] Yamashita T, Yamazaki H, Kon Y, et al. Progressive effect of alpha-phenyl-N-tert-butyl nitrone (PBN) on rat embryo development in vitro. Free Radic Biol Med 1997; 23(7): 1073-7.

[63] Morales H, Tilquin P, Rees JF, et al. Pyruvate prevents peroxideinduced injury of in vitro preimplantation bovine embryos. Mol Reprod Dev 1999; 52(2): 149-57.

[64] de Lamirande E, Leclerc P, Gagnon C. Capacitation as a regulatory event that primes spermatozoa for the acrosome reaction and fertilization. Mol Hum Reprod 1997; 3(3): 175-94.

[65] Aitken RJ, Clarkson JS, Fishel S. Generation of reactive oxygen species, lipid peroxidation, and human sperm function. Biol Reprod 1989; 41(1): 183-97.

[66] Aitken RJ, Paterson M, Fisher H, et al. Redox regulation of tyrosine phosphorylation in human spermatozoa and its role in the control of human sperm function. J Cell Sci 1995; 108 (5): $2017-$ 25 .

[67] de Lamirande E, Harakat A, Gagnon C. Human sperm capacitation induced by biological fluids and progesterone, but not by NADH or $\mathrm{NADPH}$, is associated with the production of superoxide anion. $\mathrm{J}$ Androl 1998; 19(2): 215-25.

[68] McLeod J. The role of oxygen in the metabolism and motility of human spermatozoa. Am J Physiol 1943; 138: 512-8.

[69] Aitken RJ, Clarkson JS. Cellular basis of defective sperm function and its association with the genesis of reactive oxygen species by human spermatozoa. J Reprod Fertil 1987; 81(2): 459-69.

[70] Iwasaki A, Gagnon C. Formation of reactive oxygen species in spermatozoa of infertile patients. Fertil Steril 1992; 57(2): 409-16.

[71] Mazzilli F, Rossi T, Marchesini M, et al. Superoxide anion in human semen related to seminal parameters and clinical aspects. Fertil Steril 1994; 62(4): 862-8.

[72] Sukcharoen N, Keith J, Irvine DS, et al. Prediction of the in-vitro fertilization (IVF) potential of human spermatozoa using sperm function tests: the effect of the delay between testing and IVF. Hum Reprod 1996; 11(5): 1030-4.

[73] Cummins JM, Jequier AM, Kan R. Molecular biology of human male infertility: links with aging, mitochondrial genetics, and oxidative stress? Mol Reprod Dev 1994; 37(3): 345-62.

[74] Aitken RJ, West KM, Buckingham DW. Relationship between biochemical markers for residual sperm cytoplasm reactive oxygen species generation and the presence of leucocyte and precursor germ cells in human sperm suspension. Mol Reprod Dev 1994; 39: 268.

[75] Aitken RJ, Buckingham D, West K, et al. Differential contribution of leucocytes and spermatozoa to the generation of reactive oxygen 
species in the ejaculates of oligozoospermic patients and fertile donors. J Reprod Fertil 1992; 94(2): 451-62.

[76] Kessopoulou E, Tomlinson MJ, Barratt CL, et al. Origin of reactive oxygen species in human semen: spermatozoa or leucocytes? J Reprod Fertil 1992; 94(2): 463-70.

[77] Whittington K, Ford WC. The effect of incubation periods under $95 \%$ oxygen on the stimulated acrosome reaction and motility of human spermatozoa. Mol Hum Reprod 1998; 4(11): 1053-7.

[78] Whittington K, Ford WC. Relative contribution of leukocytes and of spermatozoa to reactive oxygen species production in human sperm suspensions. Int J Androl 1999; 22(4): 229-35.

[79] Alvarez JG, Touchstone JC, Blasco L, et al. Spontaneous lipid peroxidation and production of hydrogen peroxide and superoxide in human spermatozoa. Superoxide dismutase as major enzyme protectant against oxygen toxicity. J Androl 1987; 8(5): 338-48.

[80] Alvarez JG, Storey BT. Role of glutathione peroxidase in protecting mammalian spermatozoa from loss of motility caused by spontaneous lipid peroxidation. Gamete Res 1989; 23(1): 77-90.

[81] Aitken RJ. Reactive oxygen species and human sperm function. In: Bacetti B, Ed. Comparative Spermatology 20 years after. Serono Series. New York, USA: Raven Press 1991; vol. 75: pp. 787-92.

[82] Sanocka D, Miesel R, Jedrzejczak P, et al. Oxidative stress and male infertility. J Androl 1996; 17(4): 449-54.

[83] Therond P, Auger J, Legrand A, et al. alpha-Tocopherol in human spermatozoa and seminal plasma: relationships with motility, antioxidant enzymes and leukocytes. Mol Hum Reprod 1996; 2(10): 739-44.

[84] Lewis SE, Sterling ES, Young IS, et al. Comparison of individual antioxidants of sperm and seminal plasma in fertile and infertile men. Fertil Steril 1997; 67(1): 142-7.
[85] Ford WC, Whittington K, Williams AC. Reactive oxygen species in human sperm suspensions: production by leukocytes and the generation of NADPH to protect sperm against their effects. Int J Androl 1997; 20(3): 44-9.

[86] Storey BT. Biochemistry of the induction and prevention of lipoperoxidative damage in human spermatozoa. Mol Hum Reprod 1997; 3(3): 203-13.

[87] Pasqualotto EB, Agarwal A, Sharma RK, et al. Effect of oxidative stress in follicular fluid on the outcome of assisted reproductive procedures. Fertil Steril 2004; 81(4): 973-6.

[88] Oyawoye O, Gadir AA, Garner A, et al. Antioxidants and reactive oxygen species in follicular fluid of women undergoing IVF: relationship to outcome. Hum Reprod 2003; 18(11): 2270-4.

[89] Van Blerkom J, Antczak M, Schrader R. The developmental potential of the human oocyte is related to the dissolved oxygen content of follicular fluid: association with vascular endothelial growth factor levels and perifollicular blood flow characteristics. Hum Reprod 1997; 12(5): 1047-55.

[90] Wiener-Megnazi Z, Vardi L, Lissak A, et al. Oxidative stress indices in follicular fluid as measured by the thermochemiluminescence assay correlate with outcome parameters in in vitro fertilization. Fertil Steril 2004; 82 (3): 11716.

[91] Harvey AJ, Kind KL, Thompson JG. REDOX regulation of early embryo development. Reproduction 2002; 123(4): 479-86.

[92] Burton GJ, Hempstock J, Jauniaux E. Oxygen, early embryonic metabolism and free radical-mediated embryopathies. Reprod Biomed Online 2003; 6: 84-96.

[93] Lane M, Maybach JM, Gardner DK. Addition of ascorbate during cryopreservation stimulates subsequent embryo development. Hum Reprod 2002; 17(10): 2686-93.

(C) da Silva et al.; Licensee Bentham Open.

This is an open access article licensed under the terms of the Creative Commons Attribution Non-Commercial License (http://creativecommons.org/licenses/by-nc/ $3.0 /$ ) which permits unrestricted, non-commercial use, distribution and reproduction in any medium, provided the work is properly cited. 